\title{
EMPREGO SETORIAL E O ENFRENTAMENTO À COVID-19
}

André Rauen ${ }^{1}$

Bianca Paiva ${ }^{2}$

Rafael Leão ${ }^{3}$

Bernardo Furtado ${ }^{4}$

\section{SINOPSE}

Considerando a necessidade de proteger o emprego e priorizar o apoio público, o objetivo deste trabalho é fornecer orientação à ação emergencial do Estado em nível setorial. O que este texto demonstrou foi que, entre aquelas atividades econômicas que mais empregam em todo o país, o comércio e os serviços de baixa complexidade seriam potencialmente os mais vulneráveis. Portanto, essas atividades poderiam ser priorizadas no apoio público.

Palavras-chave: pandemia; coronavírus; Covid-19; ajuda emergencial; atividades econômicas.

\section{INTRODUÇÃO}

Este trabalho tem por objetivo propor um indicador de identificação de atividades econômicas prioritárias para eventual apoio público governamental diante da atual pandemia da Covid-19 (Sars-CoV-2).

Considerando a necessidade de proteger o emprego e priorizar o apoio público, essa identificação setorial está baseada no pessoal ocupado (PO), disponibilizado na Relação Anual de Informaçôes Sociais (Rais) de 2018, último ano disponível. Assim, os dados referem-se apenas aos empregos formais no setor privado. Adicionalmente, utiliza-se como definição de atividades econômicas a desagregaçâo por grupos da Classificação Nacional das Atividades Econômicas (CNAE) versão 2.0.5

O objetivo final deste exercício empírico é o de fornecer alguma orientação à ação emergencial do Estado em todas as esferas governamentais, mesmo que ainda seja necessário algum refinamento com dados primários.

\section{MÉTODO}

O ranking de grupos econômicos prioritários (CNAE 2.0 a 3 dígitos) foi construído considerando quatro fatores, com o intuito de abranger aspectos relevantes no caso de ajuda econômica prioritária, otimizando eventuais recursos aportados:

\footnotetext{
1. Diretor na Diretoria de Estudos e Políticas Setoriais de Inovação e Infraestrutura (Diset) do Ipea.

2. Pesquisadora na Diset//pea.

3. Especialista em políticas públicas e gestão governamental na Diset/lpea e editor deste Radar.

4. Técnico de planejamento e pesquisa na Diset/lpea.

5. Disponível em: <https://bit.ly/3b9UPGZ>.
} 
- o PO do grupo perante o total da Rais;

- o coeficiente locacional do PO (Cruz et al., 2011); ${ }^{6}$

- o número de empregados acima de 60 anos no PO do grupo; ${ }^{7} \mathrm{e}$

- o número de empresas com PO menor que cinquenta empregados (uma medida de tamanho).

Essas escolhas têm por objetivo priorizar grupos econômicos de alta participação no emprego privado nacional, com alta dispersão espacial, com alta participação de idosos e empregados por empresas menores.

Para construir o ranking de grupos econômicos, diversos métodos foram testados - alguns, inclusive, com maior sofisticação. Contudo, os resultados foram sempre muito semelhantes, possivelmente porque o tamanho absoluto do PO influencia de forma direta quase todos os indicadores. Na tentativa de fornecer um indicador de fácil interpretação e rápida reprodução, optou-se por uma fórmula elaborada a partir da média simples dos números índices que compóem cada indicador individualmente, com ponderação três vezes maior para a participação setorial no PO total da Rais. Ou seja:

$$
I=\frac{(3 *(\% P O)+(\% \text { idosos })+(\% \text { pequenas })+(1-C L))}{6} .
$$

De fato, a participação no PO total é o principal elemento de definição do ranking, seja pela ponderação, seja pela presença do próprio PO nos outros indicadores individuais. Ou seja, trata-se de um ranking baseado, quase que totalmente, na participação do emprego setorial no qual os elementos adicionais apenas dão uma sintonia fina à análise. Como não se trata de uma análise de correlação, tal endogeneidade não apresenta qualquer efeito deletério à análise.

Com o ranking finalizado, foi feita uma indicação de atividades cujo funcionamento foi diretamente afetado pelos decretos de distanciamento social dos diferentes estados brasileiros. Como esses decretos variam e têm recebido constante atualização, optou-se por não incorporar tal informação na elaboração do ranking em si. Seria muito difícil afirmar que um determinado grupo econômico não possui impacto negativo mesmo tendo seu funcionamento autorizado. Nesse sentido, optou-se, apenas, por apontar os grupos afetados pela maioria dos decretos estaduais.

Adicionalmente, com o objetivo de ilustrar as implicaçôes do ranking proposto, foram também observadas as atividades profissionais - segundo a Classificação Brasileira de Ocupaçôes $(\mathrm{CBO})^{8}$ - com maior participação percentual dentro de cada atividade econômica.

6.

$E_{k i}=$ pessoal ocupado (emprego) no Grupo CNAE $k$ e Microrregião $i$.

$E_{k}=$ pessoal ocupado na Microrregião $i$.

$E_{k}=$ pessoal ocupado no Grupo CNAE $k$.

$E=$ pessoal ocupado total.

$$
C L_{k}=\frac{1}{2} \sum_{i}\left|\frac{E_{k i}}{E_{k}}-\frac{E_{i}}{E}\right| .
$$




\section{RESULTADOS}

A tabela 1 apresenta os vinte grupos econômicos prioritários com indicação da proibição ou não de fechamento, segundo os decretos de distanciamento social dos estados. Como era esperado, nenhum dos vinte primeiros é da manufatura. Tais grupos pertencem às atividades comerciais e de serviços, com especial destaque para o comércio varejista, restaurantes, serviços de baixa intensidade tecnológica e transporte de passageiros. Do ponto de vista da atividade profissional, os vendedores de comércio varejista são aqueles mais relevantes no conjunto das atividades aqui apresentadas, pois, dos vinte grupos de atividades econômicas listadas, eles aparecem em seis com o maior percentual diante de outras profissóes $\mathrm{O}$ que não significa dizer que estes profissionais serão os únicos ou mais afetados pela atual situação. Tal identificação serve apenas para ilustrar que as atividades econômicas exploradas pelas empresas têm estreita ligação com as atividades profissionais dos empregados, e isso precisa ser levado em consideração.

Mediante o exposto, conclui-se que as atividades de comércio varejista em geral, incluindo restaurantes e aqueles serviços de baixa complexidade, como serviços de mecânicos e de transporte de passageiros, são, entre os maiores empregadores brasileiros, aqueles mais vulneráveis perante a crise econômica devido à pandemia, de acordo com os critérios elencados. Portanto, essas atividades poderiam ser priorizadas no apoio público.

É evidente, contudo, que toda a economia brasileira será negativamente impactada pela pandemia provocada pela Covid-19, inclusive a manufatura. O que este texto demonstrou foi que, entre aquelas atividades econômicas que mais empregam em todo o país, o comércio e os serviços de baixa complexidade seriam potencialmente os mais vulneráveis. 


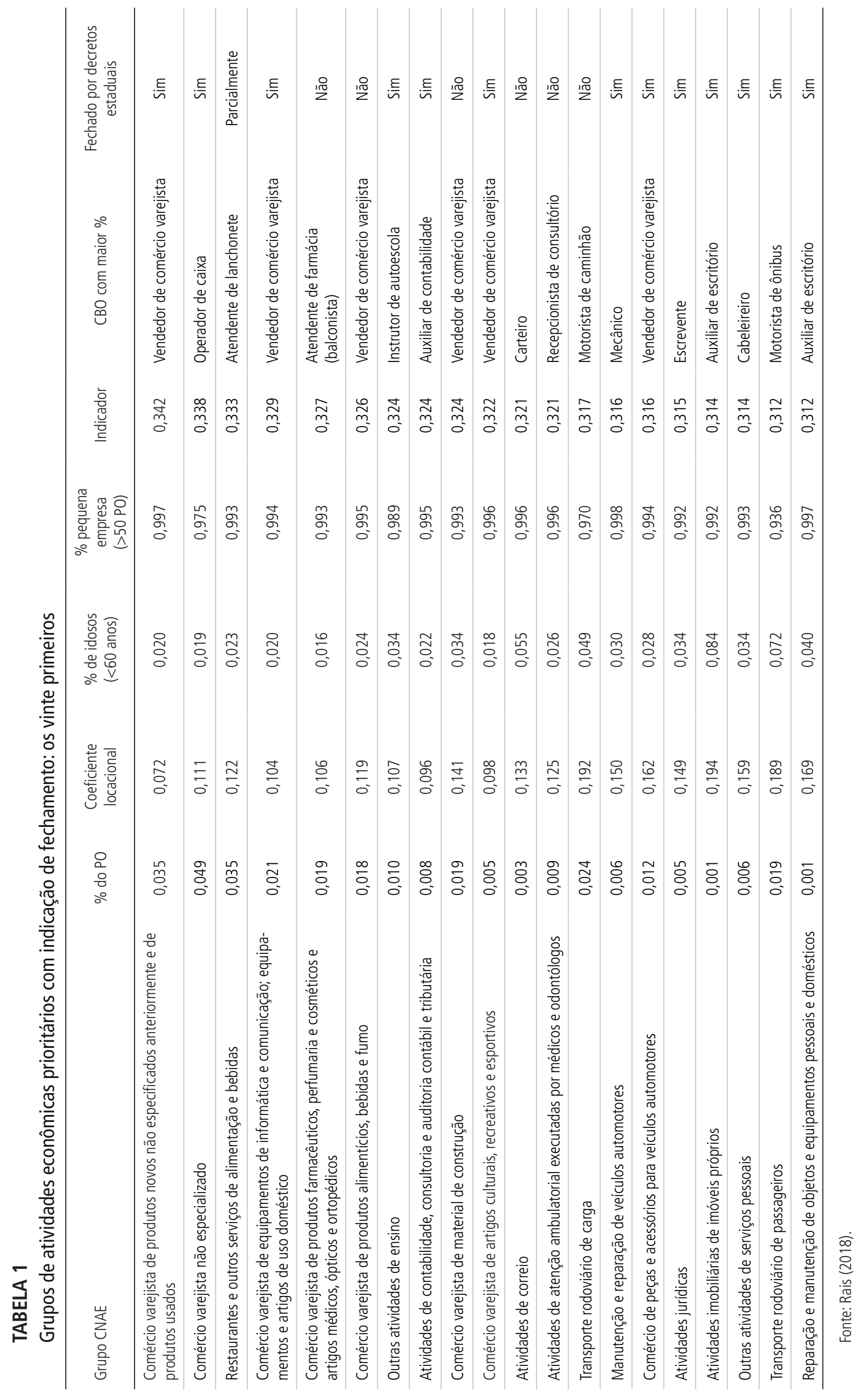




\section{REFERÊNCIAS}

CONHEÇA a faixa etária dos mortos por Covid-19 no Brasil, Itália, Espanha e EUA. Portal360, 3 abr. 2020. Disponível em: <https://bit.ly/2Rxu9Zd>. Acesso em: 14 abr. 2020.

CRUZ, B. et al. Economia regional e urbana: teorias e métodos com ênfase no Brasil. Brasília: Ipea, 2011. 


\section{APÊNDICE A}

TABELA A.1

Ranking de grupos CNAE 2.0, segundo elementos selecionados - Brasil (2018)

\begin{tabular}{|c|c|c|c|c|c|c|c|}
\hline Grupos & $\%$ do PO & Coeficiente locacional & $\%$ idosos & $\%$ tamanho & Indicador & CBO 2002 & $\%$ CBO no setor \\
\hline 478 & 0,0353 & 0,0721 & 0,0197 & 0,9965 & 0,3416 & 521110 & 0,4138 \\
\hline 471 & 0,0486 & 0,1110 & 0,0192 & 0,9745 & 0,3381 & 421125 & 0,1912 \\
\hline 475 & 0,0214 & 0,1044 & 0,0199 & 0,9942 & 0,3290 & 521110 & 0,3107 \\
\hline 477 & 0,0187 & 0,1058 & 0,0156 & 0,9932 & 0,3265 & 521130 & 0,1934 \\
\hline 859 & 0,0096 & 0,1068 & 0,0340 & 0,9887 & 0,3241 & 333105 & 0,1136 \\
\hline 692 & 0,0079 & 0,0961 & 0,0218 & 0,9946 & 0,3240 & 413110 & 0,2486 \\
\hline 474 & 0,0192 & 0,1410 & 0,0338 & 0,9934 & 0,3240 & 521110 & 0,2058 \\
\hline 476 & 0,0052 & 0,0984 & 0,0176 & 0,9956 & 0,3217 & 521110 & 0,3224 \\
\hline 531 & 0,0032 & 0,1330 & 0,0552 & 0,9958 & 0,3213 & 415205 & 0,4611 \\
\hline 452 & 0,0055 & 0,1500 & 0,0304 & 0,9979 & 0,3158 & 914405 & 0,1969 \\
\hline 453 & 0,0116 & 0,1616 & 0,0275 & 0,9938 & 0,3158 & 521110 & 0,1434 \\
\hline 691 & 0,0045 & 0,1486 & 0,0339 & 0,9915 & 0,3151 & 351405 & 0,1844 \\
\hline 681 & 0,0012 & 0,1942 & 0,0839 & 0,9918 & 0,3142 & 411005 & 0,1064 \\
\hline 960 & 0,0056 & 0,1593 & 0,0340 & 0,9928 & 0,3141 & 516110 & 0,0930 \\
\hline 492 & 0,0187 & 0,1892 & 0,0720 & 0,9361 & 0,3125 & 782410 & 0,3310 \\
\hline 952 & 0,0012 & 0,1689 & 0,0402 & 0,9974 & 0,3120 & 411005 & 0,0672 \\
\hline 949 & 0,0070 & 0,1930 & 0,0760 & 0,9676 & 0,3120 & 514320 & 0,1122 \\
\hline 931 & 0,0054 & 0,1808 & 0,0529 & 0,9809 & 0,3115 & 224120 & 0,1321 \\
\hline 750 & 0,0004 & 0,1733 & 0,0147 & 0,9978 & 0,3067 & 422105 & 0,1515 \\
\hline 468 & 0,0057 & 0,1899 & 0,0365 & 0,9685 & 0,3054 & 782510 & 0,0849 \\
\hline 943 & 0,0094 & 0,2073 & 0,0567 & 0,9546 & 0,3054 & 411005 & 0,1290 \\
\hline 772 & 0,0008 & 0,2099 & 0,0434 & 0,9950 & 0,3051 & 521110 & 0,1075 \\
\hline 851 & 0,0195 & 0,1683 & 0,0335 & 0,9066 & 0,3050 & 231210 & 0,0798 \\
\hline 829 & 0,0114 & 0,2144 & 0,0290 & 0,9750 & 0,3040 & 514320 & 0,0738 \\
\hline 181 & 0,0021 & 0,1995 & 0,0320 & 0,9818 & 0,3035 & 766315 & 0,0996 \\
\hline 451 & 0,0061 & 0,1521 & 0,0291 & 0,9242 & 0,3032 & 521110 & 0,2245 \\
\hline 773 & 0,0030 & 0,2062 & 0,0369 & 0,9796 & 0,3032 & 782510 & 0,0594 \\
\hline 467 & 0,0029 & 0,1920 & 0,0316 & 0,9685 & 0,3028 & 521110 & 0,0904 \\
\hline 682 & 0,0029 & 0,2171 & 0,0352 & 0,9865 & 0,3022 & 411005 & 0,2035 \\
\hline 951 & 0,0014 & 0,2013 & 0,0173 & 0,9918 & 0,3020 & 313220 & 0,1058 \\
\hline
\end{tabular}


(Continuação)

\begin{tabular}{|c|c|c|c|c|c|c|c|}
\hline Grupos & $\%$ do PO & Coeficiente locacional & $\%$ idosos & $\%$ tamanho & Indicador & CBO 2002 & $\%$ CBO no setor \\
\hline 562 & 0,0077 & 0,2428 & 0,0358 & 0,9789 & 0,2992 & 513505 & 0,2369 \\
\hline 461 & 0,0014 & 0,2311 & 0,0256 & 0,9934 & 0,2987 & 411005 & 0,1178 \\
\hline 942 & 0,0027 & 0,2898 & 0,0867 & 0,9750 & 0,2967 & 783210 & 0,2963 \\
\hline 865 & 0,0021 & 0,2543 & 0,0333 & 0,9947 & 0,2967 & 422105 & 0,1442 \\
\hline 433 & 0,0030 & 0,2535 & 0,0379 & 0,9854 & 0,2965 & 716610 & 0,1743 \\
\hline 109 & 0,0108 & 0,2440 & 0,0228 & 0,9665 & 0,2963 & 784205 & 0,1636 \\
\hline 662 & 0,0017 & 0,2426 & 0,0199 & 0,9935 & 0,2960 & 411005 & 0,2212 \\
\hline 331 & 0,0038 & 0,2548 & 0,0361 & 0,9813 & 0,2957 & 911305 & 0,0632 \\
\hline 941 & 0,0014 & 0,2919 & 0,0874 & 0,9718 & 0,2953 & 411010 & 0,1706 \\
\hline 464 & 0,0095 & 0,2468 & 0,0206 & 0,9689 & 0,2952 & 521110 & 0,0850 \\
\hline 432 & 0,0075 & 0,2683 & 0,0341 & 0,9781 & 0,2944 & 715615 & 0,0784 \\
\hline 611 & 0,0022 & 0,2336 & 0,0132 & 0,9767 & 0,2938 & 732130 & 0,0869 \\
\hline 791 & 0,0017 & 0,2590 & 0,0278 & 0,9882 & 0,2937 & 354815 & 0,1805 \\
\hline 932 & 0,0010 & 0,2661 & 0,0425 & 0,9818 & 0,2935 & 371410 & 0,0855 \\
\hline 821 & 0,0129 & 0,2786 & 0,0259 & 0,9748 & 0,2935 & 411010 & 0,0936 \\
\hline 871 & 0,0021 & 0,2479 & 0,0439 & 0,9574 & 0,2933 & 322205 & 0,1911 \\
\hline 551 & 0,0085 & 0,2784 & 0,0384 & 0,9712 & 0,2928 & 513315 & 0,2070 \\
\hline 823 & 0,0013 & 0,2728 & 0,0287 & 0,9905 & 0,2917 & 521115 & 0,0775 \\
\hline 411 & 0,0033 & 0,2878 & 0,0588 & 0,9664 & 0,2912 & 717020 & 0,2389 \\
\hline 582 & 0,0011 & 0,2739 & 0,0544 & 0,9614 & 0,2909 & 766315 & 0,0788 \\
\hline 601 & 0,0010 & 0,3247 & 0,0861 & 0,9809 & 0,2909 & 261715 & 0,2520 \\
\hline 233 & 0,0027 & 0,2863 & 0,0439 & 0,9781 & 0,2906 & 717020 & 0,0986 \\
\hline 742 & 0,0004 & 0,2718 & 0,0184 & 0,9944 & 0,2904 & 411010 & 0,1158 \\
\hline 522 & 0,0036 & 0,2908 & 0,0471 & 0,9751 & 0,2904 & 514110 & 0,2065 \\
\hline 771 & 0,0014 & 0,2867 & 0,0487 & 0,9755 & 0,2903 & 782305 & 0,1280 \\
\hline 813 & 0,0007 & 0,3052 & 0,0593 & 0,9800 & 0,2894 & 622010 & 0,2476 \\
\hline 332 & 0,0010 & 0,2863 & 0,0303 & 0,9858 & 0,2888 & 774105 & 0,0870 \\
\hline 466 & 0,0032 & 0,2791 & 0,0302 & 0,9696 & 0,2884 & 521110 & 0,0932 \\
\hline 251 & 0,0029 & 0,2943 & 0,0350 & 0,9791 & 0,2881 & 724440 & 0,1500 \\
\hline 591 & 0,0007 & 0,2764 & 0,0232 & 0,9791 & 0,2880 & 421115 & 0,0917 \\
\hline 649 & 0,0006 & 0,2929 & 0,0379 & 0,9788 & 0,2876 & 524105 & 0,1747 \\
\hline 711 & 0,0056 & 0,3115 & 0,0490 & 0,9664 & 0,2868 & 717020 & 0,0753 \\
\hline 532 & 0,0012 & 0,2808 & 0,0220 & 0,9754 & 0,2867 & 519110 & 0,3945 \\
\hline 900 & 0,0004 & 0,3113 & 0,0350 & 0,9946 & 0,2866 & 411010 & 0,0728 \\
\hline 431 & 0,0021 & 0,3163 & 0,0611 & 0,9652 & 0,2860 & 782510 & 0,1312 \\
\hline 619 & 0,0015 & 0,2956 & 0,0144 & 0,9698 & 0,2822 & 732130 & 0,0860 \\
\hline 454 & 0,0020 & 0,3116 & 0,0116 & 0,9864 & 0,2821 & 521110 & 0,2294 \\
\hline 731 & 0,0026 & 0,3071 & 0,0132 & 0,9783 & 0,2821 & 521115 & 0,2085 \\
\hline 639 & 0,0003 & 0,3224 & 0,0260 & 0,9864 & 0,2818 & 411005 & 0,0968 \\
\hline 329 & 0,0014 & 0,3222 & 0,0286 & 0,9753 & 0,2810 & 784205 & 0,1891 \\
\hline 295 & 0,0003 & 0,3682 & 0,0575 & 0,9957 & 0,2810 & 914405 & 0,2642 \\
\hline 854 & 0,0009 & 0,3043 & 0,0413 & 0,9458 & 0,2809 & 232115 & 0,2240 \\
\hline 182 & 0,0006 & 0,3321 & 0,0249 & 0,9876 & 0,2804 & 766315 & 0,0643 \\
\hline 559 & 0,0005 & 0,3603 & 0,0433 & 0,9972 & 0,2803 & 513315 & 0,1667 \\
\hline
\end{tabular}


(Continuação)

\begin{tabular}{|c|c|c|c|c|c|c|c|}
\hline Grupos & $\%$ do PO & Coeficiente locacional & $\%$ idosos & $\%$ tamanho & Indicador & CBO 2002 & $\%$ CBO no setor \\
\hline 429 & 0,0044 & 0,3200 & 0,0519 & 0,9291 & 0,2790 & 717020 & 0,1331 \\
\hline 381 & 0,0035 & 0,3192 & 0,0644 & 0,9164 & 0,2787 & 514205 & 0,2033 \\
\hline 873 & 0,0007 & 0,3175 & 0,0618 & 0,9216 & 0,2780 & 514320 & 0,0665 \\
\hline 469 & 0,0043 & 0,2905 & 0,0207 & 0,9223 & 0,2776 & 521125 & 0,0838 \\
\hline 861 & 0,0297 & 0,1757 & 0,0328 & 0,7145 & 0,2768 & 322205 & 0,2660 \\
\hline 253 & 0,0020 & 0,3669 & 0,0366 & 0,9762 & 0,2753 & 784205 & 0,1164 \\
\hline 970 & 0,0001 & 0,4110 & 0,0612 & 0,9935 & 0,2740 & 621005 & 0,1321 \\
\hline 880 & 0,0039 & 0,2621 & 0,0332 & 0,8604 & 0,2739 & 411005 & 0,1843 \\
\hline 620 & 0,0097 & 0,3654 & 0,0222 & 0,9439 & 0,2717 & 212405 & 0,1987 \\
\hline 712 & 0,0007 & 0,3582 & 0,0254 & 0,9556 & 0,2708 & 391205 & 0,1120 \\
\hline 383 & 0,0008 & 0,3892 & 0,0387 & 0,9710 & 0,2705 & 784205 & 0,1497 \\
\hline 799 & 0,0001 & 0,4036 & 0,0384 & 0,9878 & 0,2705 & 421120 & 0,1138 \\
\hline 421 & 0,0056 & 0,3064 & 0,0692 & 0,8424 & 0,2704 & 717020 & 0,1730 \\
\hline 360 & 0,0028 & 0,3866 & 0,1171 & 0,8830 & 0,2703 & 862205 & 0,1262 \\
\hline 812 & 0,0205 & 0,3432 & 0,0551 & 0,8481 & 0,2702 & 514320 & 0,4411 \\
\hline 325 & 0,0016 & 0,3561 & 0,0217 & 0,9506 & 0,2702 & 784205 & 0,1501 \\
\hline 646 & 0,0012 & 0,3953 & 0,0459 & 0,9651 & 0,2699 & 411010 & 0,1105 \\
\hline 81 & 0,0016 & 0,4397 & 0,0670 & 0,9540 & 0,2643 & 782510 & 0,1121 \\
\hline 141 & 0,0147 & 0,4474 & 0,0271 & 0,9604 & 0,2640 & 763210 & 0,2110 \\
\hline 592 & 0,0001 & 0,4654 & 0,0472 & 0,9959 & 0,2630 & 411005 & 0,0886 \\
\hline 702 & 0,0027 & 0,4252 & 0,0229 & 0,9690 & 0,2625 & 411010 & 0,1079 \\
\hline 869 & 0,0017 & 0,4376 & 0,0389 & 0,9685 & 0,2625 & 322205 & 0,1298 \\
\hline 222 & 0,0086 & 0,3598 & 0,0230 & 0,8849 & 0,2623 & 784205 & 0,2017 \\
\hline 310 & 0,0064 & 0,4470 & 0,0299 & 0,9643 & 0,2611 & 771105 & 0,1593 \\
\hline 152 & 0,0006 & 0,4576 & 0,0401 & 0,9769 & 0,2602 & 784205 & 0,1020 \\
\hline 422 & 0,0058 & 0,3350 & 0,0315 & 0,8462 & 0,2600 & 717020 & 0,0823 \\
\hline 852 & 0,0044 & 0,2229 & 0,0480 & 0,7175 & 0,2593 & 231210 & 0,0721 \\
\hline 465 & 0,0010 & 0,4190 & 0,0155 & 0,9564 & 0,2593 & 521110 & 0,0711 \\
\hline 351 & 0,0033 & 0,3630 & 0,0504 & 0,8560 & 0,2589 & 732120 & 0,1069 \\
\hline 221 & 0,0025 & 0,3929 & 0,0279 & 0,9103 & 0,2588 & 784205 & 0,1646 \\
\hline 282 & 0,0026 & 0,4198 & 0,0406 & 0,9216 & 0,2584 & 784205 & 0,0756 \\
\hline 661 & 0,0012 & 0,4304 & 0,0125 & 0,9476 & 0,2555 & 411010 & 0,0908 \\
\hline 853 & 0,0119 & 0,1971 & 0,0747 & 0,6117 & 0,2541 & 234520 & 0,0898 \\
\hline
\end{tabular}


(Continuação)

\begin{tabular}{|c|c|c|c|c|c|c|c|}
\hline Grupos & $\%$ do PO & Coeficiente locacional & $\%$ idosos & $\%$ tamanho & Indicador & CBO 2002 & $\%$ CBO no setor \\
\hline 525 & 0,0017 & 0,4336 & 0,0182 & 0,9351 & 0,2541 & 782220 & 0,0702 \\
\hline 370 & 0,0003 & 0,4739 & 0,0477 & 0,9492 & 0,2540 & 782510 & 0,1002 \\
\hline 721 & 0,0010 & 0,4732 & 0,1218 & 0,8647 & 0,2527 & 411010 & 0,0736 \\
\hline 872 & 0,0004 & 0,4751 & 0,0453 & 0,9430 & 0,2524 & 322205 & 0,1452 \\
\hline 207 & 0,0008 & 0,4238 & 0,0286 & 0,9055 & 0,2521 & 811130 & 0,1012 \\
\hline 323 & 0,0001 & 0,5101 & 0,0357 & 0,9822 & 0,2514 & 784205 & 0,1440 \\
\hline 286 & 0,0019 & 0,4709 & 0,0441 & 0,9255 & 0,2507 & 724315 & 0,0655 \\
\hline 174 & 0,0013 & 0,4400 & 0,0216 & 0,9175 & 0,2505 & 784205 & 0,1674 \\
\hline 774 & 0,0003 & 0,4713 & 0,0133 & 0,9595 & 0,2504 & 411010 & 0,1038 \\
\hline 862 & 0,0003 & 0,4567 & 0,0356 & 0,9173 & 0,2495 & 515135 & 0,1952 \\
\hline 642 & 0,0139 & 0,1757 & 0,0229 & 0,6013 & 0,2484 & 413225 & 0,2600 \\
\hline 231 & 0,0011 & 0,4068 & 0,0215 & 0,8693 & 0,2479 & 784205 & 0,1579 \\
\hline 910 & 0,0001 & 0,5198 & 0,0488 & 0,9537 & 0,2472 & 352205 & 0,1821 \\
\hline 655 & 0,0026 & 0,2208 & 0,0206 & 0,6747 & 0,2471 & 411010 & 0,1862 \\
\hline 106 & 0,0039 & 0,4515 & 0,0293 & 0,8911 & 0,2468 & 784205 & 0,1640 \\
\hline 284 & 0,0004 & 0,5292 & 0,0441 & 0,9634 & 0,2466 & 721215 & 0,0800 \\
\hline 801 & 0,0151 & 0,3594 & 0,0252 & 0,7546 & 0,2443 & 517330 & 0,8567 \\
\hline 15 & 0,0140 & 0,5957 & 0,0581 & 0,9594 & 0,2440 & 621005 & 0,3173 \\
\hline 462 & 0,0021 & 0,5390 & 0,0303 & 0,9647 & 0,2437 & 411005 & 0,0832 \\
\hline 135 & 0,0030 & 0,5185 & 0,0308 & 0,9342 & 0,2426 & 784205 & 0,1107 \\
\hline 16 & 0,0037 & 0,5855 & 0,0572 & 0,9657 & 0,2414 & 621005 & 0,1801 \\
\hline 209 & 0,0016 & 0,4482 & 0,0329 & 0,8557 & 0,2409 & 784205 & 0,1144 \\
\hline 162 & 0,0027 & 0,5651 & 0,0398 & 0,9598 & 0,2404 & 784205 & 0,2475 \\
\hline 279 & 0,0007 & 0,4996 & 0,0256 & 0,9144 & 0,2404 & 784205 & 0,1382 \\
\hline 822 & 0,0123 & 0,4211 & 0,0055 & 0,8207 & 0,2403 & 422310 & 0,5316 \\
\hline 602 & 0,0014 & 0,3564 & 0,0436 & 0,7476 & 0,2398 & 373130 & 0,0929 \\
\hline 105 & 0,0032 & 0,5362 & 0,0228 & 0,9318 & 0,2380 & 841505 & 0,1726 \\
\hline 920 & 0,0000 & 0,6985 & 0,1293 & 0,9916 & 0,2371 & 421205 & 0,1262 \\
\hline 111 & 0,0014 & 0,5495 & 0,0252 & 0,9387 & 0,2364 & 813125 & 0,1661 \\
\hline 234 & 0,0036 & 0,5610 & 0,0368 & 0,9310 & 0,2363 & 828110 & 0,2068 \\
\hline 12 & 0,0013 & 0,6048 & 0,0417 & 0,9752 & 0,2360 & 621005 & 0,1912 \\
\hline 803 & 0,0000 & 0,6378 & 0,0500 & 1,0000 & 0,2354 & 411005 & 0,1440 \\
\hline 112 & 0,0018 & 0,4617 & 0,0234 & 0,8442 & 0,2352 & 784205 & 0,1237 \\
\hline 265 & 0,0005 & 0,5295 & 0,0282 & 0,9105 & 0,2351 & 784205 & 0,0629 \\
\hline 173 & 0,0017 & 0,5101 & 0,0257 & 0,8886 & 0,2349 & 784205 & 0,2033 \\
\hline 273 & 0,0013 & 0,5046 & 0,0257 & 0,8785 & 0,2339 & 784205 & 0,1066 \\
\hline 103 & 0,0014 & 0,5609 & 0,0259 & 0,9344 & 0,2339 & 784205 & 0,2405 \\
\hline 108 & 0,0005 & 0,5933 & 0,0387 & 0,9556 & 0,2338 & 784205 & 0,0975 \\
\hline 274 & 0,0003 & 0,5725 & 0,0345 & 0,9321 & 0,2325 & 784205 & 0,1603 \\
\hline 321 & 0,0005 & 0,6070 & 0,0240 & 0,9739 & 0,2321 & 751125 & 0,1894 \\
\hline 732 & 0,0002 & 0,5787 & 0,0184 & 0,9458 & 0,2310 & 424115 & 0,1257 \\
\hline 293 & 0,0011 & 0,6124 & 0,0294 & 0,9605 & 0,2301 & 724315 & 0,1382 \\
\hline 524 & 0,0012 & 0,5329 & 0,0289 & 0,8807 & 0,2300 & 783205 & 0,1578 \\
\hline 244 & 0,0011 & 0,5628 & 0,0301 & 0,9062 & 0,2295 & 784205 & 0,1480 \\
\hline
\end{tabular}


(Continuação)

\begin{tabular}{|c|c|c|c|c|c|c|c|}
\hline Grupos & $\%$ do PO & Coeficiente locacional & $\%$ idosos & $\%$ tamanho & Indicador & CBO 2002 & $\%$ CBO no setor \\
\hline 781 & 0,0025 & 0,5144 & 0,0506 & 0,8298 & 0,2289 & 514320 & 0,2019 \\
\hline 183 & 0,0000 & 0,6417 & 0,0283 & 0,9810 & 0,2280 & 212405 & 0,0637 \\
\hline 511 & 0,0016 & 0,4777 & 0,0254 & 0,8079 & 0,2267 & 511105 & 0,1937 \\
\hline 390 & 0,0000 & 0,5713 & 0,0213 & 0,9077 & 0,2263 & 514225 & 0,2284 \\
\hline 855 & 0,0011 & 0,6273 & 0,0601 & 0,9054 & 0,2236 & 411010 & 0,0970 \\
\hline 281 & 0,0014 & 0,5524 & 0,0289 & 0,8533 & 0,2224 & 784205 & 0,0553 \\
\hline 651 & 0,0013 & 0,5232 & 0,0145 & 0,8354 & 0,2217 & 411010 & 0,0787 \\
\hline 134 & 0,0010 & 0,6610 & 0,0315 & 0,9423 & 0,2193 & 761410 & 0,0684 \\
\hline 201 & 0,0013 & 0,5226 & 0,0352 & 0,7977 & 0,2190 & 784205 & 0,1067 \\
\hline 324 & 0,0003 & 0,6327 & 0,0264 & 0,9075 & 0,2170 & 784205 & 0,2530 \\
\hline 161 & 0,0017 & 0,7204 & 0,0478 & 0,9688 & 0,2169 & 784205 & 0,1916 \\
\hline 11 & 0,0114 & 0,7096 & 0,0494 & 0,9226 & 0,2161 & 621005 & 0,1596 \\
\hline 203 & 0,0003 & 0,5696 & 0,0337 & 0,8306 & 0,2159 & 784205 & 0,0789 \\
\hline 322 & 0,0000 & 0,7120 & 0,0528 & 0,9508 & 0,2153 & 784205 & 0,1555 \\
\hline 261 & 0,0008 & 0,6061 & 0,0150 & 0,8802 & 0,2153 & 731180 & 0,1520 \\
\hline 266 & 0,0001 & 0,6323 & 0,0220 & 0,8972 & 0,2146 & 784205 & 0,0914 \\
\hline 654 & 0,0003 & 0,5601 & 0,0501 & 0,7881 & 0,2132 & 411010 & 0,2068 \\
\hline 99 & 0,0001 & 0,7111 & 0,0540 & 0,9346 & 0,2130 & 782510 & 0,0710 \\
\hline 142 & 0,0007 & 0,7447 & 0,0301 & 0,9677 & 0,2092 & 784205 & 0,1277 \\
\hline 643 & 0,0005 & 0,4756 & 0,0307 & 0,6971 & 0,2090 & 253225 & 0,1135 \\
\hline 512 & 0,0000 & 0,6189 & 0,0637 & 0,8077 & 0,2088 & 215305 & 0,1234 \\
\hline 382 & 0,0009 & 0,6503 & 0,1049 & 0,7929 & 0,2083 & 514215 & 0,4354 \\
\hline 294 & 0,0070 & 0,5469 & 0,0173 & 0,7559 & 0,2079 & 784205 & 0,1531 \\
\hline 509 & 0,0002 & 0,7725 & 0,0699 & 0,9457 & 0,2073 & 782705 & 0,2222 \\
\hline 101 & 0,0133 & 0,6313 & 0,0196 & 0,8111 & 0,2065 & 784205 & 0,2119 \\
\hline 271 & 0,0008 & 0,6680 & 0,0179 & 0,8760 & 0,2047 & 784205 & 0,1166 \\
\hline 283 & 0,0020 & 0,6854 & 0,0299 & 0,8773 & 0,2046 & 724315 & 0,1046 \\
\hline 32 & 0,0003 & 0,7618 & 0,0247 & 0,9633 & 0,2045 & 631325 & 0,1234 \\
\hline 31 & 0,0002 & 0,8172 & 0,0943 & 0,9455 & 0,2039 & 631210 & 0,4184 \\
\hline 513 & 0,0000 & 0,9782 & 0,2011 & 1,0000 & 0,2038 & 411005 & 0,4857 \\
\hline 14 & 0,0005 & 0,7322 & 0,0250 & 0,9199 & 0,2024 & 622115 & 0,1444 \\
\hline 491 & 0,0019 & 0,5531 & 0,0640 & 0,6961 & 0,2021 & 342405 & 0,1213 \\
\hline 352 & 0,0001 & 0,5085 & 0,0415 & 0,6780 & 0,2019 & 252105 & 0,0874 \\
\hline 242 & 0,0021 & 0,6608 & 0,0147 & 0,8500 & 0,2017 & 911305 & 0,0992 \\
\hline
\end{tabular}


(Continuação)

\begin{tabular}{|c|c|c|c|c|c|c|c|}
\hline Grupos & $\%$ do PO & Coeficiente locacional & $\%$ idosos & $\%$ tamanho & Indicador & CBO 2002 & $\%$ CBO no setor \\
\hline 285 & 0,0007 & 0,6576 & 0,0232 & 0,8400 & 0,2013 & 724315 & 0,0664 \\
\hline 22 & 0,0002 & 0,8135 & 0,0413 & 0,9776 & 0,2010 & 632325 & 0,0886 \\
\hline 614 & 0,0001 & 0,6886 & 0,0135 & 0,8797 & 0,2008 & 411005 & 0,0726 \\
\hline 211 & 0,0001 & 0,6236 & 0,0235 & 0,7979 & 0,1997 & 515225 & 0,0742 \\
\hline 133 & 0,0007 & 0,6841 & 0,0288 & 0,8511 & 0,1996 & 761330 & 0,1054 \\
\hline 132 & 0,0012 & 0,6902 & 0,0347 & 0,8488 & 0,1995 & 761005 & 0,1238 \\
\hline 89 & 0,0006 & 0,7423 & 0,0477 & 0,8874 & 0,1991 & 784205 & 0,0597 \\
\hline 268 & 0,0000 & 0,8432 & 0,0361 & 1,0000 & 0,1988 & 519110 & 0,2434 \\
\hline 13 & 0,0078 & 0,7695 & 0,0540 & 0,8825 & 0,1984 & 621005 & 0,2310 \\
\hline 243 & 0,0003 & 0,6364 & 0,0270 & 0,7977 & 0,1982 & 784205 & 0,1232 \\
\hline 192 & 0,0008 & 0,6442 & 0,0499 & 0,7805 & 0,1981 & 811310 & 0,1633 \\
\hline 262 & 0,0009 & 0,6781 & 0,0089 & 0,8544 & 0,1980 & 731180 & 0,2618 \\
\hline 501 & 0,0002 & 0,7945 & 0,1341 & 0,8431 & 0,1972 & 782705 & 0,1123 \\
\hline 301 & 0,0006 & 0,7683 & 0,0589 & 0,8889 & 0,1969 & 724210 & 0,0876 \\
\hline 641 & 0,0000 & 0,8710 & 0,0415 & 1,0000 & 0,1951 & 252545 & 0,8122 \\
\hline 267 & 0,0000 & 0,7741 & 0,0244 & 0,9146 & 0,1942 & 731150 & 0,1199 \\
\hline 204 & 0,0001 & 0,7357 & 0,0364 & 0,8636 & 0,1941 & 761005 & 0,1375 \\
\hline 783 & 0,0046 & 0,4838 & 0,0476 & 0,5827 & 0,1934 & 514320 & 0,1378 \\
\hline 104 & 0,0009 & 0,6564 & 0,0327 & 0,7774 & 0,1927 & 862150 & 0,0888 \\
\hline 263 & 0,0004 & 0,7161 & 0,0169 & 0,8542 & 0,1927 & 731180 & 0,0884 \\
\hline 309 & 0,0006 & 0,7568 & 0,0163 & 0,8918 & 0,1922 & 725505 & 0,1241 \\
\hline 23 & 0,0009 & 0,8019 & 0,0330 & 0,9183 & 0,1920 & 632125 & 0,3066 \\
\hline 264 & 0,0003 & 0,7280 & 0,0158 & 0,8593 & 0,1913 & 731180 & 0,1199 \\
\hline 275 & 0,0013 & 0,6763 & 0,0145 & 0,8049 & 0,1911 & 731175 & 0,1653 \\
\hline 523 & 0,0013 & 0,7355 & 0,0579 & 0,8195 & 0,1909 & 411010 & 0,0805 \\
\hline 122 & 0,0002 & 0,8081 & 0,0510 & 0,8931 & 0,1894 & 842125 & 0,1270 \\
\hline 502 & 0,0003 & 0,8657 & 0,0840 & 0,9055 & 0,1875 & 782705 & 0,1202 \\
\hline 653 & 0,0000 & 0,7952 & 0,0342 & 0,8800 & 0,1865 & 351735 & 0,1492 \\
\hline 131 & 0,0011 & 0,6927 & 0,0375 & 0,7631 & 0,1852 & 761005 & 0,1462 \\
\hline 102 & 0,0005 & 0,7658 & 0,0371 & 0,8310 & 0,1840 & 841484 & 0,3310 \\
\hline 153 & 0,0073 & 0,8324 & 0,0140 & 0,8977 & 0,1835 & 764005 & 0,2922 \\
\hline 644 & 0,0000 & 0,8529 & 0,0256 & 0,9231 & 0,1826 & 411010 & 0,1640 \\
\hline 212 & 0,0026 & 0,5324 & 0,0165 & 0,5814 & 0,1789 & 354150 & 0,1103 \\
\hline 647 & 0,0000 & 0,9348 & 0,0000 & 1,0000 & 0,1775 & 111415 & 0,3681 \\
\hline 503 & 0,0005 & 0,8001 & 0,0744 & 0,7881 & 0,1773 & 782705 & 0,1715 \\
\hline 154 & 0,0006 & 0,8855 & 0,0206 & 0,9207 & 0,1763 & 764005 & 0,1413 \\
\hline 303 & 0,0001 & 0,7184 & 0,0393 & 0,7273 & 0,1748 & 724315 & 0,1302 \\
\hline 495 & 0,0000 & 0,9590 & 0,0687 & 0,9375 & 0,1745 & 421125 & 0,1163 \\
\hline 353 & 0,0000 & 0,7719 & 0,0340 & 0,7826 & 0,1741 & 862120 & 0,2582 \\
\hline 60 & 0,0006 & 0,8503 & 0,0377 & 0,7973 & 0,1644 & 811310 & 0,1523 \\
\hline 494 & 0,0001 & 0,6231 & 0,0498 & 0,5556 & 0,1638 & 811005 & 0,2442 \\
\hline 72 & 0,0009 & 0,8908 & 0,0223 & 0,8407 & 0,1625 & 711130 & 0,0875 \\
\hline 151 & 0,0009 & 0,7971 & 0,0385 & 0,7227 & 0,1611 & 762005 & 0,2043 \\
\hline 652 & 0,0002 & 0,6975 & 0,0137 & 0,6471 & 0,1606 & 142330 & 0,1332 \\
\hline
\end{tabular}


Radar | 62 | abr. 2020

(Continuação)

\begin{tabular}{|c|c|c|c|c|c|c|c|}
\hline Grupos & $\%$ do PO & Coeficiente locacional & $\%$ idosos & $\%$ tamanho & Indicador & CBO 2002 & $\%$ CBO no setor \\
\hline 645 & 0,0000 & 0,6746 & 0,0382 & 0,5833 & 0,1578 & 411010 & 0,0884 \\
\hline 272 & 0,0003 & 0,8438 & 0,0162 & 0,7671 & 0,1567 & 784205 & 0,3481 \\
\hline 291 & 0,0023 & 0,6667 & 0,0058 & 0,5349 & 0,1468 & 725505 & 0,3180 \\
\hline 172 & 0,0012 & 0,7418 & 0,0277 & 0,5702 & 0,1433 & 784205 & 0,0989 \\
\hline 71 & 0,0013 & 0,8881 & 0,0129 & 0,6727 & 0,1336 & 715125 & 0,1652 \\
\hline 171 & 0,0005 & 0,8757 & 0,0280 & 0,6176 & 0,1286 & 642005 & 0,0611 \\
\hline 241 & 0,0005 & 0,8748 & 0,0400 & 0,5903 & 0,1262 & 821205 & 0,0892 \\
\hline 91 & 0,0006 & 0,8812 & 0,0220 & 0,6080 & 0,1251 & 711325 & 0,0889 \\
\hline 121 & 0,0003 & 0,9884 & 0,0701 & 0,5357 & 0,1030 & 842120 & 0,4139 \\
\hline 232 & 0,0004 & 0,7439 & 0,0313 & 0,3261 & 0,1024 & 911305 & 0,0977 \\
\hline 292 & 0,0006 & 0,7843 & 0,0128 & 0,3636 & 0,0990 & 725505 & 0,0994 \\
\hline 193 & 0,0031 & 0,8763 & 0,0362 & 0,3952 & 0,0941 & 622110 & 0,1485 \\
\hline
\end{tabular}

Elaboração dos autores.

Obs.: CNAE - Classificação Nacional das Atividades Econômicas; PO - pessoal ocupado; e CBO - Classificação Brasileira de Ocupações. 